\title{
Uninorm trust propagation and aggregation methods for group decision making in social network with four tuple information
}

\author{
Jian $\mathrm{Wu}^{\mathrm{a}, \mathrm{b}}$, Ruoyun Xiong ${ }^{\mathrm{a}}$, Francisco Chiclana ${ }^{\mathrm{b}}$ \\ ${ }^{a}$ School of Economics and Management, Zhejiang Normal University, Jinhua, Zhejiang, China \\ ${ }^{b}$ Centre for Computational Intelligence, Faculty of Technology, De Montfort University, Leicester, UK
}

\begin{abstract}
A novel social network based group decision making (SN-GDM) model with experts' weights not provided beforehand and with the following four tuple information: trust; distrust; hesitancy; and inconsistency, is introduced. The concepts of trust score (TS) and knowledge degree (KD) are defined and combined into a trust order space. Then, a strict trust ranking order relation of trust function values (TFs) is built in which TS and KD play a similar role to the mean and the variance in Statistics. After the operational laws of TFs for uninorm operators are built, the uninorm propagation operator is investigated. It can propagate through a network both trust and distrust information simultaneously and therefore it prevents the loss of trust information in the propagating process. When an indirect trust relationship is built, the uninorm trust weighted average (UTWA) operator and the uninorm trust ordered weighted average (UTOWA) operator are defined and used to aggregate individual trust relationship and to obtain their associated ranking order relation. Hence, the most trusted expert is distinguished from the group, and the weights of experts are determined in a reasonable way: the higher an expert is trusted the more importance value is assigned to the expert. Therefore, the novelty of the proposed SN-GDM is that it can use indirect trust relationship via trusted third partners (TTPs) as a reliable resource to determine experts' weights. Finally, the individual trust decision making matrices are aggregated into a collective one and the alternative with the highest trust order relation is selected as the best one.
\end{abstract}

Keywords: Group decision making, Social network, Trust propagation, Trust aggregation, Four tuple information

\section{Introduction}

In classical group decision making (GDM) problems, a group of experts express crisp preference values on alternatives under multiple criteria, and then aggregate them into a collective one for deriving a final solution [24]. However, problems with imprecise and vague information cannot be handled appropriately with crisp preferences. Atanassov's intuitionistic fuzzy set (IFS) [3], which describes each element in a set by using a membership degree $\mu_{A}(x)$ and a non-membership degree $\nu_{A}(x)$, has been

Email addresses: jyajian@163.com (Jian Wu), hixiaoxiong@sina.com (Ruoyun Xiong), chiclana@dmu.ac.uk (Francisco Chiclana) 
proved a useful tool to deal with vagueness as evidenced by the current rise of interest on its use within the research community $[31,33,34,37,42,46]$. When the membership degree and non-membership degree sum is less than one, the value $\tau_{A}(x)=1-\mu_{A}(x)-\nu_{A}(x)$ is interpreted as the hesitancy degree of experts with respect to their own judgement. Therefore, IFSs can be effectively used to model GDM problems with the following three tuple information: membership degree, non-membership degree and hesitancy degree. Apart from the hesitancy information, experts may provide inconsistent information $[6,28]$, which in this context happens when the membership degree and non-membership degree sum exceeds one. The concepts of trust and distrust have been used to model inconsistent information in [28], and to describe this heterogeneity information this paper unifies them with the concept of trust function, which is based on the following four tuple information: trust, distrust, hesitancy and inconsistency. The main reason for using trust and distrust to replace membership degree and nonmembership degree is that they are suitable to model propagating behaviour to build relationship in social network under GDM. Therefore, it can be concluded that there exist four tuple information and that dealing with this type of information representation in GDM problems is novel and not reported up to now in the literatures on the topic.

Nowadays, a new trend of GDM is that individuals rely on the opinions and social appraisal support from their close friends or people with similar interests [2]. An example is the community review website Ciao (www.ciao.com) where users can write reviews about consumer products and assign a rating to the products and the reviews written by others. In essence, Ciao is a social network, i.e. an association of people drawn together by family, work or hobby, that allows for the relationships between social entities like members of a group, corporations or nations to be studied [16, 27, 32]. By reviewing and talking, consumers can build a trust relationship between them by trust or distrust. Therefore, this group decision making under social network (SN-GDM) is of special relevance in decision contexts where the information on the problem at hand is not amenable to be modelled in a quantitative and precise way, but using four tuple information. One key issue of SN-GDM to be addressed is how to deal with hesitancy or inconsistency of information. To do that, this article first proposes the trust decision making space based on the definition of trust function (TF) [30]. Thus, according to this trust space, the definition of trust score (TS) and knowledge degree (KD) associated to trust function values (TFs) are investigated and some properties are provided. Combining TS and $\mathrm{KD}$, a ranking order relation of TFs is proposed for deriving a final solution in SN-GDM with four tuple information.

Another key issue in SN-GDM problems is how to aggregate individual preferences into a collective one for deriving a final solution. This is achieved by determining weights (or importance degrees) for each expert. In GDM models, it is often assumed that experts' weights are known beforehand or provided by a reliable source, and consequently they do not pose any challenging issue in the decision model design [5, 17, 43-45]. For example, consensus degrees have been used to assign weights to 
experts [11-14, 36, 39-41, 51]. However, SN-GDM has historical interaction relationship between members of a group, which is completely different from the classical GDM model. Thus, within the SN-GDM framework it could be interesting to provide new reliable sources to acquire experts' weight information. Recently, Herrera-Viedma et al. [18] pointed out that trust relationships between networked experts are considered as a new reliable source, while $\mathrm{Wu}$ and Chiclana [35] developed an interval-valued fuzzy social network analysis methodology to model trust relationship between experts and to compute the trust degree of each expert. This method assumed that all networked experts have direct trust relationship with other experts. Obvioulsy, in some realistic cases, these assumptions may be implausible because some experts do not have direct interactions with others, and consequently there is a need to construct indirect trust relationships between experts by using trusted third partners (TTPs), i.e. an indirect trust propagation methodology.

In order to achieve this objective, the uninorm trust propagation operator is investigated. Its properties are closely related to the properties of $t$-norm and $t$-conorm operators. However, uninorm operators can propagate trust and distrust information simultaneously and then maintain four tuple trust information in the propagating process, which is not possible to achieve with $t$-norm and $t$-conorm operators because they do not allow for trade off mechanisms of aggregation operators when fusing values in opposite side of the scale of measurement used. Thus, indirect trust relationships between any two experts are possible to be built and the propagation of the associated trust/distrust information will be carried using the uninorm trust weighted average (UTWA) operator and the uninorm trust ordered weighted average (UTOWA) operator, which are developed in this paper. Finally, the order relation of TFs can be used to distinguish the most trusted expert from the group, and is used to determine experts' weights using the rule: "the higher the expert's trust value is the higher the expert's importance value should be."

The rest of paper is set out as follows: Section 2 introduces the concepts of trust function (TF), trust decision making space (TDMS), trust score (TS) and knowledge degree (KD), which are combined to propose a ranking order relation of trust function values (TFs). Section 3 extends the uninorm operator to an $n$-dimensional space and several operation laws of TFs for uninorm operator are studied. It also investigates the indirect trust relationship propagation using the uninorm trust propagation operator. Furthermore, the UTWA and UTOWA operators are developed to aggregate propagated TFs. An illustrative example is used to verify the effect of the proposed method in Section 4. An analysis of the proposed SN-GDM with respect to the existing models in literatures is given in Section

5. Finally, conclusions are drawn in Section 6.

\section{Ranking order relationship and uninorm based operators for TFs}

The basic component of the theory of fuzzy sets (FSs) proposed by Zadeh [49] to model vagueness is the membership function, which has been argued to have its limitation when applied to decision 
contexts where it is required to deal with "a proposition A, we can state that either A is true, or A is false, or that we do not know whether A is true or false" [4]. The Intuitionistic Fuzzy Set (IFS) as introduced by Atanassov [3], which extend Zadeh's FS, is a useful mathematical concept to deal with such decision contexts:

Definition 1 (Intuitionistic fuzzy set (IFS)). An intuitionistic fuzzy set (IFS) $A$ over a universe of discourse $X$ is given by

$$
A=\left\{\left\langle x, \mu_{A}(x), \nu_{A}(x)\right\rangle \mid x \in X\right\}
$$

where

$$
\mu_{A}: X \rightarrow[0,1], \nu_{A}: X \rightarrow[0,1]
$$

and

$$
0 \leq \mu_{A}(x)+\nu_{A}(x) \leq 1 \quad \forall x \in X
$$

For each element $x$, the values $\mu_{A}(x)$ and $\nu_{A}(x)$ represent the degree of membership and the degree of non-membership of $x$ to $A$, respectively.

An IFS becomes a FS when $\mu_{A}(x)=1-\nu_{A}(x) \forall x \in X$. However, when there exists at least an element $x \in X$ for which $\mu_{A}(x)<1-\nu_{A}(x)$, an extra parameter is to be taken into account when working with IFSs, the hesitancy degree, $\tau_{A}(x)$, of $x$ to $A$ :

$$
\tau_{A}(x)=1-\mu_{A}(x)-\nu_{A}(x)
$$

The hesitancy degree $\tau_{A}(x)$ is an indicator of the hesitation margin of the membership of element $x$ to the IFS $A$ and therefore it represents the amount of lacking information in determining the membership of $x$ to $A$. The above three values, which add one, in relation to the concept of membership can be considered as positive/favourable $\left(\mu_{A}(x)\right)$, negative/unfavourable $\left(\nu_{A}(x)\right)$, and hesitation $\left(\tau_{A}(x)\right)$, respectively.

\subsection{Trust function and trust degree}

In some real cases, experts may have inconsistency decision making information due to them not possessing a sufficient level of knowledge of the problem or to their non-rationality human nature, which in the above context of IFSs formally happen when $\mu_{A}(x)+\nu_{A}(x)>1$. In order to accommodate inconsistency with IFSs, the concept of trust decision making space is proposed, which is based on the following concept of trust function with trust degree and distrust degree given by Victor et al. [30]:

Definition 2 (Trust Function (TF)). A tuple of the type $\lambda=(t, d)$ where $t, d \in[0,1]$, in which the first component $t$ is a trust degree, and the second component $d$ is a distrust degree will be referred to as a trust function value. The set of trust function values (TFs), or trust function, will be denoted by $\Lambda=\{\lambda=(t, d) \mid t, d \in[0,1]\} \equiv[0,1]^{2}$. 
Using the above definition of trust function and equation (2) as discussed above, a trust decision making space can be defined to describe the possible different types of decision making information:

Definition 3 (Trust Decision Making Space (TDMS)). The trust decision making space consists of the following three elements: the set of TFs, $\Lambda$, a trust hesitancy space, THS, and a trust inconsistency space $T C S$, i.e.

$$
T D M S^{\square}=(\Lambda, T H S, T C S)
$$

with

$$
T H S=\{\lambda \in \Lambda \mid t+d \leq 1\}
$$

and

$$
T C S=\{\lambda \in \Lambda \mid t+d>1\}
$$

Alternatives in decision making problems can therefore be evaluated using four types of information: trust, distrust, hesitancy and inconsistency. Selection of alternatives will be done by using a new ranking method for TFs to be proposed later in the paper. First, the trust score and knowledge degree associated to TFs are presented.

Definition 4 (Trust Score). The trust score is a mapping on the set of TFs, $\Lambda$, that associates a value in $[0,1]$ to each trust function value $\lambda$ as follows:

$$
\begin{gathered}
T S: \Lambda \longrightarrow[0,1] \\
T S(\lambda)=\frac{t-d+1}{2}
\end{gathered}
$$

Because $0 \leq T S \leq 1$, it is reasonable to use it in Eq. (23) (Section 3) to determine experts' weights when using the regular increasing monotone (RIM) quantifier $Q(r)=r^{1 / 2}[23]$ driven by the rule: the higher the value of TS, the higher the experts' importance degree. Indeed, TS represents the normalised dominance that the trust value has over the corresponding distrust value of a trust function value of an expert, i.e. the strict trust value contained in a trust function. When two experts have the same trust score (TS), the uncertainty degree associated to their respective TFs as represented in the following definition can be used to further differentiate them.

Definition 5 (Knowledge Degree). The knowledge degree is a mapping on the set of TFs, $\Lambda$, that associates a value in $[0,1]$ to each trust function value $\lambda$ as follows:

$$
\begin{gathered}
K D: \Lambda \longrightarrow[0,1] \\
K D(\lambda)=(1-t-d)^{2}
\end{gathered}
$$


Notice that when information is inconsistent as for example with $(0.7,0.4)$, the concept of hesitation degree of intuitionistic fuzzy sets cannot be used, $H D=1-t-d$, to evaluate the uncertainty degree of TFs because it is a negative value. In particular, the closer KD is to zero, the closer $t+d$ will be to 1 , and therefore the closer the values will resemble the output of the representation of trust using fuzzy sets, i.e. hesitation degree in absolute value will be close to zero, and in absolute value lacking of information in determining the trust function value of an expert is low. As per [30], TFs for which $K D(\lambda)=0$, i.e. $t+d=1$, have perfect knowledge or complete trust state, otherwise there exists trust knowledge uncertainty. Thus, KD is a supplement to TS in ranking TFs.

Notice that the set $\Lambda$ is closed under the normalised weighted average operation. Indeed, given a set of weights $\left\{w_{1}, w_{2}, \ldots, w_{n} \mid w_{i} \geq 0 \wedge \sum_{i=1}^{n} w_{i}=1\right\}$ and a set of TFs $\left\{\lambda_{1}, \lambda_{2}, \ldots, \lambda_{n}\right\}$ then it is

$$
\sum_{i=1}^{n} w_{i} \cdot \lambda_{i}=\sum_{i=1}^{n} w_{i} \cdot\left(t_{i}, d_{i}\right)=\left(\sum_{i=1}^{n} w_{i} \cdot t_{i}, \sum_{i=1}^{n} w_{i} \cdot d_{i}\right) \in \Lambda .
$$

The following properties are verified by $T S$ and $K D$ :

Proposition 1. Given a set of weights $\left\{w_{1}, w_{2}, \ldots, w_{n} \mid w_{i} \geq 0 \wedge \sum_{1}^{n} w_{i}=1\right\}$ and a set of TFs $\left\{\lambda_{1}, \lambda_{2}, \ldots, \lambda_{n}\right\}$, the following properties hold:

$$
\begin{aligned}
& \text { - } T S\left(\sum_{i=1}^{n} w_{i} \cdot \lambda_{i}\right)=\sum_{i=1}^{n} w_{i} \cdot T S\left(\lambda_{i}\right) \\
& \text { - } K D\left(\sum_{i=1}^{n} w_{i} \cdot \lambda_{i}\right) \leq \sum_{i=1}^{n} w_{i} \cdot K D\left(\lambda_{i}\right)
\end{aligned}
$$

Proof. Recall that $w_{i} \geq 0(\forall i) \wedge \sum_{1}^{n} w_{i}=1$.

- The proof of the first equality is obvious because $T S$ is a linear function. Nevertheless, it is provided for completion.

$$
\begin{aligned}
T S\left(\sum_{i=1}^{n} w_{i} \cdot \lambda_{i}\right) & =\frac{\sum_{i=1}^{n} w_{i} \cdot t_{i}-\sum_{i=1}^{n} w_{i} \cdot d_{i}+1}{2}=\frac{\sum_{i=1}^{n} w_{i} \cdot t_{i}-\sum_{i=1}^{n} w_{i} \cdot d_{i}+\sum_{i=1}^{n} w_{i}}{2} \\
& =\frac{\sum_{i=1}^{n} w_{i} \cdot\left(t_{i}-d_{i}+1\right)}{2}=\sum_{i=1}^{n} w_{i} \cdot \frac{t_{i}-d_{i}+1}{2}=\sum_{i=1}^{n} w_{i} \cdot \operatorname{TS}\left(\lambda_{i}\right)
\end{aligned}
$$

- Let $a_{1}, a_{2}, \ldots, a_{n}$ and $b_{1}, b_{2}, \ldots, b_{n}$ be real numbers. Then the Cauchy-Schwarz inequality holds:

$$
\left(\sum_{i=1}^{n} a_{i} \cdot b_{i}\right)^{2} \leq \sum_{i=1}^{n} a_{i}^{2} \cdot \sum_{i=1}^{n} b_{i}^{2}
$$

Thus:

$$
\begin{aligned}
K D\left(\sum_{i=1}^{n} w_{i} \cdot \lambda_{i}\right) & =\left(1-\sum_{i=1}^{n} w_{i} \cdot t_{i}-\sum_{i=1}^{n} w_{i} \cdot d_{i}\right)^{2}=\left(\sum_{i=1}^{n} w_{i}-\sum_{i=1}^{n} w_{i} \cdot t_{i}-\sum_{i=1}^{n} w_{i} \cdot d_{i}\right)^{2} \\
& =\left(\sum_{i=1}^{n} w_{i} \cdot\left(1-t_{i}-d_{i}\right)\right)^{2}
\end{aligned}
$$


Denoting $a_{i}=\sqrt{w_{i}}$ and $b_{i}=\sqrt{w_{i}} \cdot\left(1-t_{i}-d_{i}\right)$, the Cauchy-Schwarz inequality implies that

$$
K D\left(\sum_{i=1}^{n} w_{i} \cdot \lambda_{i}\right) \leq \sum_{i=1}^{n} w_{i} \cdot \sum_{i=1}^{n} w_{i} \cdot\left(1-t_{i}-d_{i}\right)^{2}=\sum_{i=1}^{n} w_{i} \cdot K D\left(\lambda_{i}\right)
$$

Later in the paper, it will be shown that the set $\Lambda$ is closed under a new general uninorm based operation introduced to propagate trust in social networks.

\subsection{Ranking order relationship for TFs}

In a SN-GDM problem, the weights of experts according to their associated trust degrees are needed so that the highest trusted expert within their social network can be differentiated. This trust problem is influenced by the presence of hesitancy or inconsistency information. Therefore, a model should be proposed to represent appropriately, on the one hand, the trust an agent may have on another agent, and on the other hand, to allow evaluating the contribution that each aspect of trust has in the overall trust opinion [27]. Following a similar approach to the one provided in [8], TS and $\mathrm{KD}$ are combined in a trust order space as a model that allows to compare and preserve information about the provenance of TFs as follows:

Definition 6. (Trust Order Space (TOS)). A trust order space

$$
T O S^{\square}=\left(\Lambda, \leq_{T S}, \leq_{K D}, \neg\right)
$$

consists of the set of TFs $\Lambda$, a trust ordering $\leq_{T S}$, a knowledge ordering $\leq_{K D}$, and a negation operator $\neg$ that verify the following properties

$$
\begin{aligned}
& \lambda_{1} \leq_{T S} \lambda_{2} \text { iff } T S_{1} \leq T S_{2} \\
& \lambda_{1} \leq_{K D} \lambda_{2} \text { iff } K D_{1} \geq K D_{2} \\
& \neg(t, d)=(d, t)
\end{aligned}
$$

In a TOS, TSs are used to evaluate the degree of strict trust an agent may have on other agents in the social network when providing his TFs, while KDs are used to determine the uncertainty contained in the corresponding TFs. Their role for ranking TFs is similar to the mean and the variance in Statistics. In detail, TOS allows the following order relation on the set of TFs, $\Lambda$, to be defined:

Definition 7 (Order Relation of TFs). Given two TFs, $\lambda_{1}$ and $\lambda_{2}, \lambda_{1}$ precedes $\lambda_{2}$

$$
\lambda_{1} \prec \lambda_{2}
$$

if and only if one of the following conditions is true:

1. $T S\left(\lambda_{1}\right)<T S\left(\lambda_{2}\right)$ 
2. $T S\left(\lambda_{1}\right)=T S\left(\lambda_{2}\right) \wedge K D\left(\lambda_{1}\right)>K D\left(\lambda_{2}\right)$

The next result proves that the order relation $\prec$ is a strict order.

Theorem 1 (Strict Order of TFs). The relation $\prec$ on the set of TFs $\Lambda$ is:

1. Irreflexive: $\forall \lambda: \lambda \prec \lambda$ does not hold.

2. Asymmetric: $\forall \lambda_{1}, \lambda_{2}$ : if $\lambda_{1} \prec \lambda_{2}$, then $\lambda_{2} \prec \lambda_{1}$ does not hold.

3. Transitive: $\forall \lambda_{1}, \lambda_{2}, \lambda_{3}$ : if $\lambda_{1} \prec \lambda_{2}$ and $\lambda_{2} \prec \lambda_{3}$, then $\lambda_{1} \prec \lambda_{3}$.

Proof. Items 1. and 2. are obvious from Definition 7. To prove the transitivity property, assuming $\lambda_{1} \prec \lambda_{2}$, from Definition 7 there are two possible cases:

1. $T S\left(\lambda_{1}\right)<T S\left(\lambda_{2}\right)$. Because $T S\left(\lambda_{2}\right) \leq T S\left(\lambda_{3}\right)$ it is $T S\left(\lambda_{1}\right)<T S\left(\lambda_{3}\right)$ and therefore $\lambda_{1} \prec \lambda_{3}$.

2. $T S\left(\lambda_{1}\right)=T S\left(\lambda_{2}\right) \wedge K D\left(\lambda_{1}\right)>K D\left(\lambda_{2}\right)$. Because $\lambda_{2} \prec \lambda_{3}$ then one of the following is true:

(a) $T S\left(\lambda_{2}\right)<T S\left(\lambda_{3}\right)$, which implies that $T S\left(\lambda_{1}\right)<T S\left(\lambda_{3}\right)$ and consequently it is $\lambda_{1} \prec \lambda_{3}$.

(b) $T S\left(\lambda_{2}\right)=T S\left(\lambda_{3}\right) \wedge K D\left(\lambda_{2}\right)>K D\left(\lambda_{3}\right)$. Thus, $T S\left(\lambda_{1}\right)=T S\left(\lambda_{3}\right) \wedge K D\left(\lambda_{1}\right)>K D\left(\lambda_{3}\right)$, i.e. $\lambda_{1} \prec \lambda_{3}$.

The order relation of TFs can distinguish the most trusted expert from the group, and then can be used to assign weights to experts in a reasonable way, i.e. the higher the trust ranking order of an expert is, the more importance the expert will be associated. Therefore, it can deal with GDM problems without the importance information of experts known beforehand as this can be indirectly derived using their corresponding TFs.

\subsection{Uninorm based operations on TFs}

The following section will introduce the addition of TFs and the scalar multiplication of TFs based on uninorm operators and its properties. To do so, we first provide the necessary background information on the uninorm [48]:

Definition 8. A uninorm $U$ is a mapping $U:[0,1]^{2} \longrightarrow[0,1]$ having the following properties:

1. Commutativity: $U(x, y)=U(y, x)$

2. Monotonicity: $U\left(x_{1}, y_{1}\right) \geq U\left(x_{2}, y_{2}\right)$ if $x_{1} \geq x_{2}$ and $y_{1} \geq y_{2}$

3. Associativity: $U(x, U(y, z))=U(U(x, y), z)$

4. Identity element: $\exists e \in[0,1]: \forall x \in[0,1], U(x, e)=x$ 
The uninorm with t-norm and t-conorm the commutativity, associativity and monotonicity properties, and its behaviour is closely related as it is explained later. The identity element property allows to claim that the unimorm generalises both the t-norm and t-conorm, as they can be seen a particular uninorm: a t-norm is obtained when $e=1$ in Def. 8 , while a t-conorm is obtained when $e=0$. In general, a uninorm can have an identity element lying anywhere in the unit interval $[0,1]$ (see also $[21])$.

As pointed out by Fodor et al. [15], an interesting particular case of uninorm is symmetric aggregative operators, i.e. uninorms that have a representation in terms of a single variable function

$$
U(x, y)=h^{-1}(h(x)+h(y)), \quad x, y \in[0,1]
$$

where $h:[0,1] \longrightarrow \overline{\mathbb{R}}$ is a strictly increasing continuous function with $h(e)=0, h(0)=-\infty$ and $h(1)=+\infty$. Representable uninorms are strictly increasing on the open unit interval square and selfdual with respect to a strong negation: a continuous and strictly decreasing mapping $N:[0,1] \longrightarrow$ $[0,1]$ such that $N(N(x))=x(\forall x \in[0,1])$ and $N(U(x, y))=U(N(x), N(y))(\forall x, y \in[0,1])$. The representation theorem also provides a relationship between the generator function $h$ and the strong negation $N: h^{-1}(-h(x))=N(x)$. For the particular case when $N(x)=1-x$, the identity element is $e=0.5, h(x)+h(1-x)=0$ and $h(0.5)=0$. Additionally, in this case when the generator function $h(x)=\ln \frac{x}{1-x}$, it results in the well known andlike representable cross ratio uninorm [21]

$$
U(x, y)= \begin{cases}0, & (x, y) \in\{(0,1),(1,0)\} \\ \frac{x y}{x y+(1-x)(1-y)}, & \text { Otherwise }\end{cases}
$$

This particular uninorm is used in the PROSPECTOR expert system [9] and it has been characterised as the most appropriate for modelling cardinal consistency of reciprocal preference relations in $[1$, 6]. Therefore, its use in the proposed model guarantees an appropriate handling of inconsistent information in SN-GDM. From now on, the reference to a uninorm within the context of the SN-GDM model here investigated means a representable uninorm $U$ verifying $U(1,0)=U(0,1)=0$.

The behaviour of uninorms on the squares $[0,0.5] \times[0,0.5]$ and $[0.5,1] \times[0.5,1]$ is closely related to t-norms and t-conorms [15], respectively. Indeed, the cross ratio uninorm can be rewritten as follows:

- $\forall x, y \in[0,0.5]: U(x, y)=\frac{T_{U}(2 x, 2 y)}{2}$

- $\forall x, y \in[0.5,1]: U(x, y)=\frac{S_{U}(2 x-1,2 y-1)}{2}$

with $T_{U}(x, y)=\frac{x y}{1+(1-x)(1-y)}(\forall x, y \in[0,1])$ the Einstein product t-norm and $S_{U}(x, y)=$ $\frac{x+y}{1+x y}(\forall x, y \in[0,1])$ its dual Einstein sum t-conorm [7]. Definition 8 presents the uninorm as an aggregation operator of two arguments. However, the associativity property allows its use as an aggregation operator with $n$ arguments. The cross ratio uninorm with $n$ arguments has the following 
expression:

$$
U\left(x_{1}, x_{2}, \ldots, x_{n}\right)= \begin{cases}0, & \text { if } \exists i, j:\left(x_{i}, x_{j}\right) \in\{(0,1),(1,0)\} \\ \frac{\prod_{i=1}^{n} x_{i}}{\prod_{i=1}^{n} x_{i}+\prod_{i=1}^{n}\left(1-x_{i}\right)}, & \text { Otherwise. }\end{cases}
$$

Next we introduce the uninorm sum operation on the set of TFs:

Definition 9. Given a uninorm $U$, the uninorm sum operation on the set of TFs $\Lambda, \oplus_{U}: \Lambda \times \Lambda \longrightarrow \Lambda$, is given as

$$
\lambda_{1} \oplus U \lambda_{2}=\left(U\left(t_{1}, t_{2}\right), U\left(d_{1}, d_{2}\right)\right)
$$

where $\lambda_{1}=\left(t_{1}, d_{1}\right)$ and $\lambda_{2}=\left(t_{2}, d_{2}\right)$.

The cross ratio uninorm sum of two TFs yields:

$\lambda_{1} \oplus_{U} \lambda_{2}= \begin{cases}(0,0), & \text { if }\left(\lambda_{1}, \lambda_{2}\right) \in\{((0,1),(1,0)),((1,0),(0,1))\} \\ \left(\frac{t_{1} t_{2}}{t_{1} t_{2}+\left(1-t_{1}\right)\left(1-t_{2}\right)}, \frac{d_{1} d_{2}}{d_{1} d_{2}+\left(1-d_{1}\right)\left(1-d_{2}\right)}\right), & \text { Otherwise. }\end{cases}$

Uninorms are commutative and associative, and therefore the uninorm sum is also commutative and associative. The repetitive uninorm sum of a trust function value with itself results in the uninorm scalar multiplication of TFs by a natural number:

Definition 10. Given a uninorm $U$, the uninorm scalar multiplication of a trust function value $\lambda \in \Lambda$ by a natural number $n$ is given as

$$
n \odot{ }_{U} \lambda=\overbrace{\lambda \oplus_{U} \lambda \oplus_{U} \ldots \oplus_{U} \lambda}^{n}
$$

For the cross ratio uninorm, the uninorm scalar multiplication of a trust function value $\lambda=(t, d)$ by a natural number $n$ yields:

$$
n \odot_{U} \lambda=\left(\frac{t^{n}}{t^{n}+(1-t)^{n}}, \frac{d^{n}}{d^{n}+(1-d)^{n}}\right)
$$

When the uninorm is continuous, the uninorm scalar multiplication of TFs can be extended to any real number. The following proposition lists additional distributivity properties for the case of using the cross ratio uninorm in both the uninorm sum and the uninorm scalar multiplication.

Proposition 2. Let $\lambda=(t, d), \lambda_{1}=\left(t_{1}, d_{1}\right), \lambda_{2}=\left(t_{2}, d_{2}\right) \in \Lambda$ and $\delta, \delta_{1}, \delta_{2}$ be any three real numbers. The following properties are verified:

1. $\delta \odot_{U}\left(\lambda_{1} \oplus_{U} \lambda_{2}\right)=\left(\delta \odot_{U} \lambda_{1}\right) \oplus_{U}\left(\delta \odot_{U} \lambda_{2}\right)$

2. $\left(\delta_{1}+\delta_{2}\right) \odot_{U} \lambda=\left(\delta_{1} \odot_{U} \lambda\right) \oplus_{U}\left(\delta_{2} \odot_{U} \lambda\right)$ 


$$
A=\left(\begin{array}{llllll}
0 & 1 & 1 & 1 & 1 & 0 \\
0 & 0 & 0 & 0 & 1 & 0 \\
0 & 1 & 0 & 0 & 0 & 0 \\
0 & 0 & 1 & 0 & 1 & 1 \\
0 & 0 & 1 & 0 & 0 & 1 \\
0 & 0 & 1 & 0 & 0 & 0
\end{array}\right)
$$

Sociometric

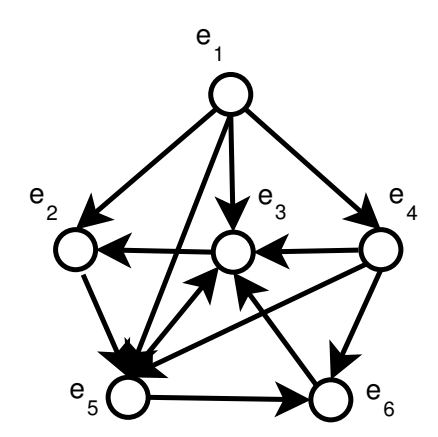

Graph $\begin{array}{ll}e_{1} R e_{2} & e_{4} R e_{3} \\ e_{1} R e_{3} & e_{4} R e_{5} \\ e_{1} R e_{4} & e_{4} R e_{6} \\ e_{1} R e_{5} & e_{5} R e_{3} \\ e_{2} R e_{5} & e_{5} R e_{6} \\ e_{3} R e_{2} & e_{6} R e_{3}\end{array}$

Algebraic

Table 1: Different notations in Social Network Analysis

3. $\left(\delta_{1} \cdot \delta_{2}\right) \odot_{U} \lambda=\delta_{1} \odot_{U}\left(\delta_{2} \odot_{U} \lambda\right)$

Proof. It is omitted.

The uninorm sum, $\oplus_{U}$, and uninorm scalar multiplication, $\odot_{U}$, will be used in the following sections to develop several uninorm trust propagation and aggregation operators in the context of social network.

\section{Uninorm trust propagation and aggregation operators}

Trust relationship has been regarded as a reliable source to evaluate the importance degree of experts [35, 38], which is usually studied by Social Network Analysis (SNA) [16, 27, 32]. There are three notational schemes in SNA analysis: set of actors, the relations themselves, and the actor criteria (see Table 1).

- Sociometric - relational data presented in a two-ways matrix called sociomatrix.

- Graph theoretic - the network is viewed as a graph consisting of nodes joined by lines.

- Algebraic - allows to distinguish several distinct relations and represent combinations of relations.

The sociomatrix given in Table 1 represents a crisp complete relation, i.e. all its elements are known and represents just the presence or absence of relationship but not its intensity, which might not be a suitable representation in social network in the presence of uncertainty of the relationship to model. Notice that in real life too, trust is indeed often interpreted as a gradual phenomenon: humans do not merely reason in terms of 'trusting' and 'not trusting', but rather trusting someone 'very much' or 'more or less' [10]. This work focuses on one type of social networks, namely trust network in which the users explicitly express their opinion as trust and distrust statements [30].

In a trust network, some experts might not have a direct trust relationship with others because they are typically unknown to them as Fig. 1 illustrates. Therefore, there is a need to devise a 


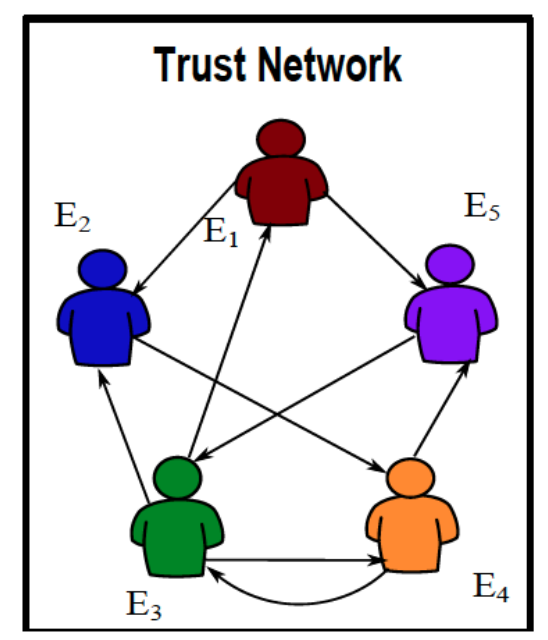

Figure 1: Graph representation of the trust network

mechanism to find out whether or not an unknown expert can be trusted. This can be achieved via an indirect chain of trusted third partners (TTPs) to propagate trust to an unknown expert. Therefore, one key issue is to develop appropriate trust propagation operators. Victor et al. [29] used t-norms to propagate trust and t-conorms to propagate distrust, respectively. However, the use of two distinct and separate operators to independently propagate information might not be best practice as trust and distrust are not independent. This issue can be overcome by using both operators together in the trust propagation, which is possible by employing a uninorm instead as the unifying propagation operator. Indeed, as we have seen before, a uninorm behaves like a t-norm when all values are below the identity element, like a t-conorm when all values are above the identity element and like a symmetric mean otherwise [26]. Therefore, a uninorm propagation operator will allow both trust and distrust to be propagated simultaneously. This is elaborated next.

\subsection{Uninorm trust propagation operator}

Figure 2(a) illustrates a typical case with three experts in which there is no direct trust function value between two of them. However, an indirect chain of TFs using another expert can be exploited to build the missing direct trust function value between these experts by propagating the corresponding known indirect TFs as Fig. 2(b) shows.

Notice that in the scenario represented in Fig. 2, on the one hand, expert $E_{1}$ trust value on expert $E_{3}$ should be based on his/her own trust value on expert $E_{2}$ and the corresponding trust value expert $E_{2}$ has on expert $E_{3}$. On the other hand, $E_{1}$ distrust value on expert $E_{3}$ should be based on the known direct distrust value on $E_{3}$, i.e the distrust value $E_{2}$ has on expert $E_{3}$, in conjunction with his/her own trust value on expert $E_{2}$. In a complete trust/distrust scenario, it is expected that when expert $E_{1}$ fully trusts $E_{2}$ and expert $E_{2}$ fully trusts $E_{3}$ then $E_{1}$ will fully trust $E_{3}$, while expert $E_{1}$ will fully distrust $E_{3}$ when expert $E_{2}$ fully distrusts $E_{3}$ and expert $E_{1}$ fully trusts $E_{2}$. This can be achieved using the following uninorm propagation operator: 

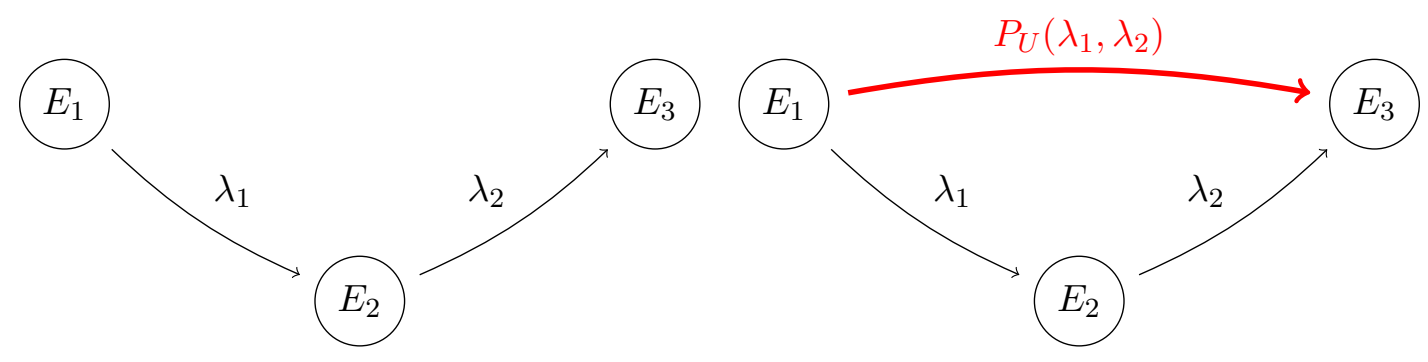

(a) No direct trust function value between $E_{1}$ and (b) Trust propagation between $E_{1}$ and $E_{3}$ via $E_{2}$ $E_{3}$

Figure 2: Uninorm trust propagation via indirect chain of TFs

Definition 11. Let $U$ be a uninorm. The uninorm trust propagation operator $P_{U}$ is the mapping, $P_{U}: \Lambda \times \Lambda \longrightarrow \Lambda$, that associates two TFs $\lambda_{1}=\left(t_{1}, d_{1}\right), \lambda_{2}=\left(t_{2}, d_{2}\right)$ with the following trust function value output

$$
P_{U}\left(\lambda_{1}, \lambda_{2}\right)=\left(U\left(t_{1}, t_{2}\right), U\left(t_{1}, d_{2}\right)\right)
$$

Notice that when $\lambda_{1}=\left(t_{1}, d_{1}\right)=(1,0)$ and $\lambda_{2}=\left(t_{2}, d_{2}\right)=(1,0)$, expert $E_{1}$ fully trusts expert $E_{2}$ and expert $E_{2}$ fully trusts expert $E_{3}$, and the application of the uninorm trust propagation operator $P_{U}$ results in $P_{U}((1,0),(1,0))=(U(1,1), U(1,0))=(1,0)$, which means that expert $E_{1}$ fully trusts expert $E_{3}$ as expected. When $\lambda_{1}=\left(t_{1}, d_{1}\right)=(1,0)$ and $\lambda_{2}=\left(t_{2}, d_{2}\right)=(0,1)$, expert $E_{1}$ fully trusts expert $E_{2}$ and expert $E_{2}$ fully distrusts $E_{3}$, and the application of the the uninorm trust propagation operator $P_{U}$ results in $P_{U}((1,0),(0,1))=(U(1,1), U(1,1))=(0,1)$, which means that expert $E_{1}$ will fully distrusts expert $E_{3}$ as it is also expected. Therefore, the uninorm trust propagation operator generalises the propagation method proposed by Victor et al. in [30].

In general, if expert $E_{1}$ fully trusts expert $E_{2}$, then the trust function value of expert $E_{1}$ on expert $E_{3}$ is expected to be the same as the trust function value of expert $E_{2}$ on expert $E_{3}$, and consequently it would be

$$
P_{U}\left(\lambda_{1}, \lambda_{2}\right)=\lambda_{2} \text { when } \lambda_{1}=(1,0)
$$

On the other hand, when expert $E_{2}$ fully trusts expert $E_{3}$ then the trust function value of expert $E_{1}$ on expert $E_{3}$ is expected to be the same as the trust function value of expert $E_{1}$ on expert $E_{2}$ and consequently it would be

$$
P_{U}\left(\lambda_{1}, \lambda_{2}\right)=\lambda_{1} \text { when } \lambda_{2}=(1,0) .
$$

What should be expected when in the above chain expert $E_{1}$ fully distrusts expert $E_{2}$ ? In this case, any trust function value coming from expert $E_{2}$ would be completely dismissed by expert $E_{1}$ and the full distrust on expert $E_{2}$ by expert $E_{1}$ would be propagated to the rest of the chain, and consequently it would be

$$
P_{U}\left(\lambda_{1}, \lambda_{2}\right)=(0,1) \text { when } \lambda_{1}=(0,1) \text {. }
$$


When $E_{2}$ fully distrusts expert $E_{3}$, no matter what trust function value there is from expert $E_{1}$ towards expert $E_{2}$, expert $E_{1}$ is expected to fully distrust expert $E_{3}$. Thus, the following representation of the uninorm trust propagation operator is proposed:

$$
P_{U}\left(\lambda_{1}, \lambda_{2}\right)= \begin{cases}\lambda_{2}, & \text { if } \lambda_{1}=(1,0) \\ \lambda_{1}, & \text { if } \lambda_{2}=(1,0) \\ (0,1), & \text { if } \lambda_{1} \vee \lambda_{2}=(0,1) \\ \left(U\left(t_{1}, t_{2}\right), U\left(t_{1}, d_{2}\right)\right), & \text { Otherwise }\end{cases}
$$

Given two TFs $\lambda_{1}=\left(t_{1}, d_{1}\right), \lambda_{2}=\left(t_{2}, d_{2}\right)$, the cross ratio uninorm trust propagation operator would be:

$$
P_{U}\left(\lambda_{1}, \lambda_{2}\right)= \begin{cases}\lambda_{2}, & \text { if } \lambda_{1}=(1,0) \\ \lambda_{1}, & \text { if } \lambda_{2}=(1,0) \\ (0,1), & \text { if } \lambda_{1} \vee \lambda_{2}=(0,1) \\ \left(\frac{t_{1} t_{2}}{t_{1} t_{2}+\left(1-t_{1}\right)\left(1-t_{2}\right)}, \frac{t_{1} d_{2}}{t_{1} d_{2}+\left(1-t_{1}\right)\left(1-d_{2}\right)}\right), & \text { Otherwise. }\end{cases}
$$

Example 1. Given the TFs $\lambda_{1}=(0.55,0.5), \lambda_{2}=(0.52,0.51)$, the cross ratio uninorm trust propagation operator gives

$$
P_{U}=(0.57,0.56)
$$

The propagating operator proposed by Victor et al. [29] gives

$$
P_{V}=(0.55 \times 0.52,0.55 \times 0.51)=(0.286,0.281)
$$

Since $\lambda_{1}$ and $\lambda_{2}$ contain inconsistent information $(t+d>1)$, then their propagating result should be of the same type, which is properly reflected by $P_{U}$ but not by $P_{V}$. Therefore, the uninorm trust propagation operator does not lose important information such as inconsistency; the same cannot be affirmed for the case of the trust propagation operator proposed by Victor et al. [29].

Notice that associativity property of the uninorm $U$ implies associativity of the propagation operator $P_{U}$, so trust can be propagated on an incomplete trust path involving more than three experts as illustrated in Fig. 3.

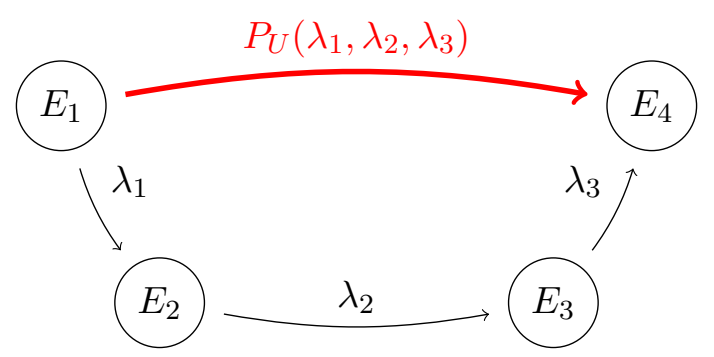

Figure 3: Trust propagation of orthopairs of trust/distrust values between $E_{1}$ and $E_{4}$ via trust path $E_{1} \rightarrow E_{2} \rightarrow E_{3} \rightarrow E_{4}$ 
Indeed, if $\lambda_{1}, \lambda_{2}, \lambda_{3} \notin\{(0,1),(1,0)\}$ then

$$
\begin{aligned}
P_{U}\left(P_{U}\left(\lambda_{1}, \lambda_{2}\right), \lambda_{3}\right) & =P_{U}\left(P_{U}\left(\left(t_{1}, d_{1}\right),\left(t_{2}, d_{2}\right)\right),\left(t_{3}, d_{3}\right)\right) \\
& =P_{U}\left(\left(U\left(t_{1}, t_{2}\right), U\left(t_{1}, d_{2}\right)\right),\left(t_{3}, d_{3}\right)\right) \\
& =\left(U\left(U\left(t_{1}, t_{2}\right), t_{3}\right), U\left(U\left(t_{1}, t_{2}\right), d_{3}\right)\right) \\
& =\left(U\left(t_{1}, U\left(t_{2}, t_{3}\right)\right), U\left(t_{1}, U\left(t_{2}, d_{3}\right)\right)\right) \\
P_{U}\left(\lambda_{1}, P_{U}\left(\lambda_{2}, \lambda_{3}\right)\right) & =P_{U}\left(\left(t_{1}, d_{1}\right), P_{U}\left(\left(t_{2}, d_{2}\right),\left(t_{3}, d_{3}\right)\right)\right) \\
& =P_{U}\left(\left(t_{1}, d_{1}\right),\left(U\left(t_{2}, t_{3}\right), U\left(t_{2}, d_{3}\right)\right)\right) \\
& =\left(U\left(t_{1}, U\left(t_{2}, t_{3}\right)\right), U\left(t_{1}, U\left(t_{2}, d_{3}\right)\right)\right)
\end{aligned}
$$

For the case when $\lambda_{1}, \lambda_{2}, \lambda_{3} \in\{(0,1),(1,0)\}$ then:

- If $\lambda_{1}=(1,0)$, on the one hand $P_{U}\left(P_{U}\left(\lambda_{1}, \lambda_{2}\right), \lambda_{3}\right)=P_{U}\left(\lambda_{2}, \lambda_{3}\right)$, and on the other hand it is $P_{U}\left(\lambda_{1}, P_{U}\left(\lambda_{2}, \lambda_{3}\right)\right)=P_{U}\left(\lambda_{2}, \lambda_{3}\right)$. In this case, it is $P_{U}\left(\lambda_{1}, \lambda_{2}, \lambda_{3}\right)=P_{U}\left(\lambda_{2}, \lambda_{3}\right)$. The cases $\lambda_{2}=(1,0)$ or $\lambda_{3}=(1,0)$ are proved similarly. Notice that having a trust function value in a chain equal to $(1,0)$ means that it can be dropped from the uninorm trust propagation operation.

- When one trust function value is equal to $(0,1)$ the final trust function value resulting from the applicatioin of the uninorm trust propagation operator would be $(0,1)$.

From now on, and to simplify expressions, it will be assumed that $\lambda_{1}, \lambda_{2}, \lambda_{3} \notin\{(0,1),(1,0)\}$ being the rest of limit cases easy to verify. It can be concluded then that

$$
P_{U}\left(\lambda_{1}, \lambda_{2}, \lambda_{3}\right)=\left(U\left(t_{1}, t_{2}, t_{3}\right), U\left(t_{1}, t_{2}, d_{3}\right)\right)
$$

Expression (14) can be extended to an arbitrary number of experts $n(\geq 3)$ as follows:

$$
P_{U}\left(\left(t_{1}, d_{1}\right),\left(t_{2}, d_{2}\right), \ldots,\left(t_{n}, d_{n}\right)\right)=\left(U\left(t_{1}, t_{2}, \ldots, t_{n}\right), U\left(t_{1}, t_{2}, \ldots, t_{n-1}, d_{n}\right)\right)
$$

To prove (15), induction on $n$ is applied:

1. Basis: $n=3$. This is the associativity property (14) already proved to be true.

2. Induction hypothesis: Let's assume that (15) is true for $n=k$ and prove that it is also true when $n=k+1$. Applying associativity of $P$ we have:

$$
P_{U}\left(\left(t_{1}, d_{1}\right),\left(t_{2}, d_{2}\right), \ldots,\left(t_{k+1}, d_{k+1}\right)\right)=P_{U}\left(P_{U}\left(\left(t_{1}, d_{1}\right),\left(t_{2}, d_{2}\right), \ldots,\left(t_{k}, d_{k}\right)\right),\left(t_{k+1}, d_{k+1}\right)\right)
$$

Applying that (15) is true for $n=k$ we have:

$$
P_{U}\left(\left(t_{1}, d_{1}\right),\left(t_{2}, d_{2}\right), \ldots,\left(t_{k+1}, d_{k+1}\right)\right)=P_{U}\left(\left(U\left(t_{1}, t_{2}, \ldots, t_{k}\right), U\left(t_{1}, t_{2}, \ldots, t_{k-1}, d_{k}\right)\right),\left(t_{k+1}, d_{k+1}\right)\right)
$$

Definition of $P_{U}$ is applied to obtain:

$$
P_{U}\left(\left(t_{1}, d_{1}\right),\left(t_{2}, d_{2}\right), \ldots,\left(t_{k+1}, d_{k+1}\right)\right)=\left(U\left(U\left(t_{1}, t_{2}, \ldots, t_{k}\right), t_{k+1}\right), U\left(U\left(t_{1}, t_{2}, \ldots, t_{k}\right), d_{k+1}\right)\right)
$$


Applying associativity of uninorm $U$ yields:

$$
P_{U}\left(\left(t_{1}, d_{1}\right),\left(t_{2}, d_{2}\right), \ldots,\left(t_{k+1}, d_{k+1}\right)\right)=\left(U\left(t_{1}, t_{2}, \ldots, t_{k+1}\right), U\left(t_{1}, t_{2}, \ldots, t_{k}, d_{k+1}\right)\right)
$$

Using the uninorm sum of TFs, the following result is proved:

Proposition 3. Let $\left\{\lambda_{j}=\left(t_{j}, d_{j}\right) \in \Lambda \mid j \in\{1,2, \ldots, n\}\right\}$ be a collection of $n(>2)$ TFs. Then, the propagated trust function value can be obtained as follows:

$$
P_{U}\left(\lambda_{1}, \lambda_{2}, \ldots, \lambda_{n}\right)=P_{U}\left(\left(\lambda_{1} \oplus_{U} \lambda_{1} \oplus_{U} \ldots \oplus_{U} \lambda_{n-1}\right), \lambda_{n}\right)
$$

Proof. Applying the definition of uninorm sum, it is:

$$
\lambda_{1} \oplus_{U} \lambda_{1} \oplus_{U} \ldots \oplus_{U} \lambda_{n-1}=\left(U\left(t_{1}, \ldots, t_{n-1}\right), U\left(d_{1}, \ldots, d_{n-1}\right)\right)
$$

Consequently

$$
P_{U}\left(\left(\lambda_{1} \oplus_{U} \lambda_{1} \oplus_{U} \ldots \oplus_{U} \lambda_{n-1}\right), \lambda_{n}\right)=P_{U}\left(\left(U\left(t_{1}, \ldots, t_{n-1}\right), U\left(d_{1}, \ldots, d_{n-1}\right)\right),\left(t_{n}, d_{n}\right)\right)
$$

Applying Definition 11, it is:

$$
P_{U}\left(\left(\lambda_{1} \oplus_{U} \lambda_{1} \oplus_{U} \ldots \oplus_{U} \lambda_{n-1}\right), \lambda_{n}\right)=\left(U\left(U\left(t_{1}, \ldots, t_{n-1}\right), t_{n}\right), U\left(U\left(t_{1}, \ldots, t_{n-1}\right), d_{n}\right)\right)
$$

This proves that:

$$
P_{U}\left(\lambda_{1}, \lambda_{2}, \ldots, \lambda_{n}\right)=P_{U}\left(\left(\lambda_{1} \oplus_{U} \lambda_{1} \oplus_{U} \ldots \oplus_{U} \lambda_{n-1}\right), \lambda_{n}\right)
$$

In this paper, the cross ratio uninorm will be used following the next two steps in succession:

1. Discard all TFs equal to $(1,0)$.

2. Otherwise, apply the following expression to the rest of TFs

$$
P_{U}\left(\lambda_{1}, \lambda_{2}, \ldots, \lambda_{n}\right)= \begin{cases}(0,1), & \exists j: \lambda_{j}=(0,1) \\ \left(\frac{\prod_{j=1}^{n} t_{j}}{\prod_{j=1}^{n} t_{j}+\prod_{j=1}^{n}\left(1-t_{j}\right)}, \frac{\left(\prod_{j=1}^{n-1} t_{j}\right) d_{n}}{\left(\prod_{j=1}^{n-1} t_{j}\right) d_{n}+\left(\prod_{j=1}^{n-1}\left(1-t_{j}\right)\right)\left(1-d_{n}\right)}\right), & \text { Otherwise. }\end{cases}
$$

Note 1. When propagating trust from one (start) node of a social network to another (end) node with no direct trust relationship, it could happen that there exist more than one indirect propagation paths between them. In these cases, it might be reasonable to use the shortest indirect path, i.e. the path with minimum number of different intermediate nodes between the start and end nodes. Also, in the proposed trust network, given the trust function value of expert $E_{i}$ on another expert $E_{j}$ being $\lambda_{i j}=\left(t_{i j}, d_{i j}\right)$, only when $T S_{i j}=t_{i j}-d_{i j}>0$ it can be confirmed that expert $E_{i}$ trusts expert $E_{j}$, and then this trust relationship can be propagated. 


\subsection{Uninorm trust weighted average (UTWA) operator}

The uninorm sum and uninorm scalar multiplication allow to formally define the uninorm trust weighted average (UTWA) operator of a set of TFs as follows:

Definition 12. Let $\left\{\lambda_{j}=\left(t_{j}, d_{j}\right) \in \Lambda \mid j \in\{1,2, \ldots, n\}\right\}$ be a collection of $n(>2)$ TFs and $W=$ $\left(w_{1}, w_{2}, \ldots, w_{n}\right)$ be a weighting vector such that $w_{j} \in[0,1]$ and $\sum_{j=1}^{n} w_{j}=1$. The uninorm trust weighted average (UTWA) is computed as:

$$
\operatorname{UTWA}_{W}\left(\lambda_{1}, \lambda_{2}, \ldots, \lambda_{n}\right)=\left(w_{1} \odot_{U} \lambda_{1}\right) \oplus_{U}\left(w_{2} \odot_{U} \lambda_{2}\right) \oplus_{U} \cdots \oplus_{U}\left(w_{n} \odot_{U} \lambda_{n}\right)
$$

The uninorm trust arithmetic average (UTAA) operator is obtained when all weights are equal to $1 / n$.

For the cross ratio uninorm, using expressions (8) and (10), the UTWA operator reduces to:

$\mathrm{UTWA}_{W}\left(\lambda_{1}, \lambda_{2}, \ldots, \lambda_{n}\right)= \begin{cases}\left(\frac{\prod_{j=1}^{n} t_{j}^{w_{j}}}{\prod_{j=1}^{n} t_{j} w_{j}+\prod_{j=1}^{n}\left(1-t_{j}\right)^{w_{j}}}, \frac{\prod_{j=1}^{n} d_{j} w_{j}}{\prod_{j=1}^{n} d_{j} w_{j}+\prod_{j=1}^{n}\left(1-d_{j}\right)^{w_{j}}}\right. & \\ (0,0), & \lambda_{j} \notin\{(0,1),(1,0)\} \\ \text { Otherwise. }\end{cases}$

The order relation of TFs given in Definition 7 allows to formally define the uninorm trust OWA (UTOWA) operator as follows:

Definition 13. Let $\left\{\lambda_{j}=\left(t_{j}, d_{j}\right) \in \Lambda \mid j \in\{1,2, \ldots, n\}\right\}$ be a collection of $n(>2)$ TFs and $W=$ $\left(w_{1}, w_{2}, \ldots, w_{n}\right)$ be a weighting vector such that $w_{j} \in[0,1]$ and $\sum_{j=1}^{n} w_{j}=1$. The uninorm trust ordered weighted average (UTOWA) is computed as:

$$
\mathrm{UTOWA}_{W}\left(\lambda_{1}, \lambda_{2}, \ldots, \lambda_{n}\right)=\left(w_{1} \odot_{U} \lambda_{\sigma(1)}\right) \oplus_{U}\left(w_{2} \odot_{U} \lambda_{\sigma(2)}\right) \oplus_{U} \cdots \oplus_{U}\left(w_{n} \odot_{U} \lambda_{\sigma(n)}\right)
$$

$\sigma:\{1, \ldots, n\} \rightarrow\{1, \ldots, n\}$ being the permutation that orders the TFs from highest to lowest: $\lambda_{\sigma(i)} \succeq$ $\lambda_{\sigma(i+1)}(\forall i \in\{1, \ldots, n-1\})$.

Similarly to the UTWA, the UTOWA expression for the cross ratio uninorm is:

$\mathrm{UTOWA}_{W}\left(\lambda_{1}, \lambda_{2}, \ldots, \lambda_{n}\right)= \begin{cases}\left(\frac{\prod_{j=1}^{n} t_{\sigma(j)} w_{j}}{\prod_{j=1}^{n} t_{\sigma(j)} w_{j}+\prod_{j=1}^{n}\left(1-t_{\sigma(j)}\right)^{w_{j}}}, \frac{\prod_{j=1}^{n} d_{\sigma(j)} w_{j}}{\prod_{j=1}^{n} d_{\sigma(j)} w_{j}+\prod_{j=1}^{n}\left(1-d_{\sigma(j)}\right)^{w_{j}}}\right), & \lambda_{j} \notin\{(0,1),(1,0)\} \\ (0,0), & \text { Otherwise. }\end{cases}$

The implementation of the UTOWA operator requires to compute beforehand its associated weighting vector. On the one hand, the sets of TFs are reordered using Definition 7, and therefore the trust scores associated to the TFs actually induce the ordering of the arguments to aggregate. On the 
other hand, the higher the trust score associated to a trust function value, the higher should be the importance associated to such trust function value. Thus, trust scores can also be implemented in deriving the weighting vector of the UTOWA operator, which can be achieved by using Yager's procedure to evaluate the overall satisfaction of a 'soft majority' $(Q)$ of important criteria (experts) by an alternative $(x)$ as follows [47]:

$$
w_{h}=Q\left(\frac{S(h)}{S(n)}\right)-Q\left(\frac{S(h-1)}{S(n)}\right)
$$

being $Q$ a Basic Unit-interval Monotone (BUM) membership function (non-decreasing $Q:[0,1] \rightarrow[0,1]$ such that $Q(0)=0, Q(1)=1$ ) of the linguistic quantifier [50] representing the concept of 'soft majority' to implement in the decision making resolution [20,25];S(h)= $\sum_{l=1}^{h} s_{\sigma(l)}, s_{l}$ the importance degree of criterion $l$; and $\sigma$ the permutation used to produce the ordering of the values to aggregate. In the present context, as mentioned above, trust scores are used to induce the ordering of the TFs but also as measure of their importance. Consequently, the weights associated to the UTOWA operator are computed as follows

$$
w_{\sigma(h)}=Q\left(\frac{T(\sigma(h))}{T(\sigma(n))}\right)-Q\left(\frac{T(\sigma(h-1))}{T(\sigma(n))}\right)
$$

with $T(\sigma(h))=\sum_{l=1}^{h} T S\left(\lambda_{\sigma(l)}\right)$, and $\lambda_{\sigma(i)} \succeq \lambda_{\sigma(i+1)}(\forall i \in\{1, \ldots, n-1\})$.

Yager [47] considered the parameterised family of regular increasing monotone (RIM) quantifiers $Q(r)=r^{a}(a \geq 0)$ for the implementation of the concept of 'soft majority'. This family of functions guarantees that: (i) all the experts contribute to the final aggregated value (strict monotonicity property), and (ii) associates, when $a \in[0,1]$, higher weight values to the aggregated values with associated higher importance values[19]. In particular, the value $a=1 / 2$ is used to represent the fuzzy linguistic quantifier 'most of'.

\section{Illustrative Example}

A person wants to buy a dust coat, with four types of dust coat to select from, $\left\{x_{1}, x_{2}, x_{3}, x_{4}\right\}$, and the following five criteria to consider: colour; comfort; style; environmental protection property; brand. The five criteria weighting vector is $W_{C}=(0.25,0.20,0.15,0.10,0.30)$. However, this person has little knowledge about dust coats and consults some of his/her socially networked friends regarded as experts [22], becoming a SN-GDM problem.

Step 1. We assume that five experts $\left\{E_{1}, E_{2}, E_{3}, E_{4}, E_{5}\right\}$ have the following trust relationship given in Figure 1 with corresponding trust sociomatrix $T_{L}$ :

$$
T_{L}=\left(\begin{array}{ccccc}
- & (0.6,0.2) & & & (0.7,0.3) \\
& - & & (0.6,0.1) & \\
(0.6,0.1) & (0.8,0.2) & - & (0.7,0.2) & \\
& & (0.6,0.3) & - & (0.6,0.3) \\
& & (0.9,0.3) & & -
\end{array}\right)
$$


This is an incomplete trust sociomatrix as there is no direct trust link between all the nodes of the network, although indirect chain of trusted third partners (TTPs) can be used to propagate trust in those cases. For example, to propagate TFs from expert $E_{1}$ to expert $E_{3}$, we have three possible indirect TTPs paths: $L_{1}: E_{1} \rightarrow E_{5} \rightarrow E_{3}$ and $L_{2}: E_{1} \rightarrow E_{2} \rightarrow E_{4} \rightarrow E_{3}$, and $L_{3}: E_{1} \rightarrow E_{2} \rightarrow$ $E_{4} \rightarrow E_{5} \rightarrow E_{3}$, and therefore $L_{1}$ is used as it is the shortest path. According to expression (17), the propagated trust function value from expert $E_{1}$ to expert $E_{3}$ is:

$$
P_{U}^{L_{1}}((0.7,0.3),(0.9,0.3))=(0.95,0.50)
$$

Applying the above same process, the completed trust sociomatrix would be:

$$
T_{L}=\left(\begin{array}{ccccc}
- & (0.60,0.20) & (0.95,0.50) & (0.78,0.39) & (0.70,0.30) \\
(0.77,0.20) & - & (0.69,0.39) & (0.60,0.10) & (0.69,0.39) \\
(0.60,0.10) & (0.80,0.20) & - & (0.70,0.20) & (0.78,0.50) \\
(0.69,0.14) & (0.86,0.27) & (0.60,0.30) & - & (0.60,0.30) \\
(0.93,0.50) & (0.97,0.69) & (0.90,0.30) & (0.95,0.69) & -
\end{array}\right)
$$

After the complete trust sociomatrix is achieved, the uninorm trust arithmetic average of each expert is computed as a measure of their importance in the network:

$$
\bar{\lambda}_{i}=U T A A\left(\lambda_{i 1}, \ldots, \lambda_{i j}, \ldots \lambda_{i n}\right)
$$

Using the cross ratio uninorm yields:

$$
\bar{\lambda}_{1}=(0.78,0.21) ; \bar{\lambda}_{2}=(0.85,0.32) ; \bar{\lambda}_{3}=(0.83,0.37) ; \bar{\lambda}_{4}=(0.80,0.31) ; \bar{\lambda}_{5}=(0.70,0.37) ;
$$

According to Definition 4, it is

$$
T D_{1}=0.785 ; T D_{2}=0.765 ; T D_{3}=0.730 ; T D_{4}=0.745 ; T D_{5}=0.665
$$

and Def. 7 gives:

$$
\bar{\lambda}_{\sigma(1)}=\bar{\lambda}_{1} ; \bar{\lambda}_{\sigma(2)}=\bar{\lambda}_{2} ; \bar{\lambda}_{\sigma(3)}=\bar{\lambda}_{4} ; \bar{\lambda}_{\sigma(4)}=\bar{\lambda}_{3} ; \bar{\lambda}_{\sigma(5)}=\bar{\lambda}_{5}
$$

The application of Eq. (23) results in the following experts' weights:

$$
W_{E}=(0.461,0.187,0.117,0.141,0.095)
$$

Step 2. It is assumed here that the group of experts provide the following trust evaluation for the four types of dust coat (rows) with respect each one of the criteria (columns):

$$
R^{1}=\left(\begin{array}{ccccc}
{[0.7,0.8]} & {[0.3,0.7]} & {[0.3,0.7]} & {[0.5,0.3]} & {[0.5,0.7]} \\
{[0.5,0.6]} & {[0.4,0.2]} & {[0.4,0.7]} & {[0.6,0.8]} & {[0.5,0.2]} \\
{[0.4,0.3]} & {[0.5,0.9]} & {[0.4,0.2]} & {[0.5,0.3]} & {[0.4,0.7]} \\
{[0.6,0.4]} & {[0.3,0.4]} & {[0.3,0.6]} & {[0.6,0.9]} & {[0.3,0.6]}
\end{array}\right)
$$




$$
\begin{array}{r}
R^{2}=\left(\begin{array}{lllll}
{[0.3,0.4]} & {[0.5,0.2]} & {[0.4,0.6]} & {[0.7,0.5]} & {[0.8,0.3]} \\
{[0.7,0.6]} & {[0.6,0.4]} & {[0.3,0.5]} & {[0.4,0.7]} & {[0.5,0.6]} \\
{[0.5,0.7]} & {[0.3,0.6]} & {[0.8,0.2]} & {[0.6,0.3]} & {[0.2,0.8]} \\
{[0.4,0.8]} & {[0.7,0.5]} & {[0.6,0.6]} & {[0.3,0.4]} & {[0.9,0.1]}
\end{array}\right) \\
R^{3}=\left(\begin{array}{lllll}
{[0.5,0.3]} & {[0.6,0.4]} & {[0.3,0.5]} & {[0.4,0.8]} & {[0.7,0.6]} \\
{[0.8,0.4]} & {[0.3,0.7]} & {[0.6,0.8]} & {[0.5,0.3]} & {[0.5,0.2]} \\
{[0.6,0.5]} & {[0.2,0.9]} & {[0.5,0.4]} & {[0.3,0.6]} & {[0.6,0.7]} \\
{[0.7,0.4]} & {[0.5,0.5]} & {[0.4,0.7]} & {[0.8,0.2]} & {[0.3,0.4]}
\end{array}\right) \\
R^{4}=\left(\begin{array}{lllll}
{[0.7,0.4]} & {[0.4,0.3]} & {[0.3,0.6]} & {[0.6,0.4]} & {[0.8,0.4]} \\
{[0.3,0.5]} & {[0.5,0.2]} & {[0.2,0.9]} & {[0.7,0.7]} & {[0.6,0.5]} \\
{[0.6,0.2]} & {[0.7,0.6]} & {[0.4,0.5]} & {[0.3,0.8]} & {[0.9,0.2]} \\
{[0.4,0.8]} & {[0.6,0.5]} & {[0.5,0.3]} & {[0.7,0.2]} & {[0.5,0.7]}
\end{array}\right) \\
R^{5}=\left(\begin{array}{lllll}
{[0.4,0.8]} & {[0.6,0.7]} & {[0.3,0.5]} & {[0.7,0.4]} & {[0.5,0.6]} \\
{[0.6,0.3]} & {[0.5,0.2]} & {[0.4,0.6]} & {[0.8,0.2]} & {[0.7,0.1]} \\
{[0.7,0.5]} & {[0.8,0.3]} & {[0.5,0.5]} & {[0.6,0.5]} & {[0.4,0.5]} \\
{[0.3,0.4]} & {[0.7,0.1]} & {[0.6,0.3]} & {[0.4,0.9]} & {[0.5,0.8]}
\end{array}\right)
\end{array}
$$

The individual trust decision making matrices are aggregated into a collective one using the UTOWA $_{W_{E}}$ operator and the experts' trust scores to induce the ordering, which results in:

$$
\bar{R}=\left(\begin{array}{ccccc}
{[0.57,0.63]} & {[0.41,0.51]} & {[0.32,0.63]} & {[0.56,0.42]} & {[0.64,0.56]} \\
{[0.56,0.53]} & {[0.45,0.28]} & {[0.37,0.71]} & {[0.59,0.66]} & {[0.54,0.28]} \\
{[0.50,0.40]} & {[0.49,0.78]} & {[0.50,0.28]} & {[0.47,0.43]} & {[0.47,0.63]} \\
{[0.51,0.55]} & {[0.48,0.40]} & {[0.42,0.54]} & {[0.57,0.69]} & {[0.48,0.50]}
\end{array}\right)
$$

Step 3. The application of the UTWA operator guided by the criteria weighting vector $W_{C}$ results in the following alternatives' TFs:

$$
\lambda_{x_{1}}=(0.52,0.56) ; \lambda_{x_{2}}=(0.51,0.44) ; \lambda_{x_{3}}=(0.49,0.54) ; \lambda_{x_{4}}=(0.49,0.52)
$$

The trust scores of alternatives are:

$$
T S\left(x_{1}\right)=0.480 ; T S\left(x_{2}\right)=0.535 ; T S\left(x_{3}\right)=0.475 ; T S\left(x_{4}\right)=0.485
$$

Thus, the order relation of the set of alternatives is

$$
x_{3} \prec x_{1} \prec x_{4} \prec x_{2}
$$

and the dust coat to select would be $x_{2}$. 
In GDM problems, IFSs have been proved a useful tool to deal with hesitancy information of experts. However, IFSs cannot describe the inconsistency of information among a group of users (agents) in a social network. Obviously, the proposed SN-GDM model is able to unify both hesitancy and inconsistency information in a trust function. For example in $R^{1}, r_{11}^{1}=[0.7,0.8]$ represents inconsistency information while $r_{13}^{1}=[0.5,0.3]$ represents hesitancy information. Furthermore, the uninorm trust propagation operator allows to exploit the trust relationship among the group of users full, which is not the case with previously proposed propagation operators. Therefore, the proposed trust relationship provide a new reliable source to derive and assign experts' weights and it is not a requirement to known these beforehand, and as a consequence the proposed SN-GDM model is a novel and suitable method to deal with more complex decision making problems than previous GDM methods.

\section{Analysis of the proposed method}

In this article, the uninorm trust propagation based SN-GDM with four tuple information has been presented. This SN-GDM model has the following main advantages with respect to other group decision making models proposed in the literatures:

1. It uses four tuple information (trust, distrust, hesitancy and inconsistency) to model individual preferences in group decision making process under social network. Therefore, it can be regarded as an extension of intuitionistic fuzzy sets [3], which just contains three tuple information (trust, distrust, hesitancy). Furthermore, it is important to remark that this model is one of the first efforts to use four tuple information to model group decision making problems.

2. It introduces some new definitions associated with trust function (TF), such as: trust score (TS) and knowledge degree (KD), and then proposes the trust decision making space. Combining TS and $\mathrm{KD}$, the ranking order relation of trust function values (TFs) is developed.

3. It investigates the uninorm trust propagation operator, which extends previous proposal based on $t$-norms and $t$-conorms. Also, it allows to propagate trust and distrust information simultaneously, and then it carries both hesitancy and inconsistency when present. Furthermore, the uninorm trust weighted average (UTWA) operator and the uninorm trust ordered weighted average (UTOWA) operator are developed to aggregate TFs between a group of experts or a set of criteria. It is worth remarking that this model is one of the first efforts to use indirect trust relationship by trusted third partners (TTPs) to determine the weights of experts, being indeed more complex and realistic than the direct one presented in [35].

4. Our previous work [38] focuses on an interactive method for consensus in group decision making problem with incomplete linguistic information. The calculation method of TFs are modified 
from [29], and then it is used to estimate the missing linguistic information in the decision making matrix and determine the weights of experts as a reliable source. Different from [38], this work aims to deal with MCGDM probelms with four tuple information. Obviously, the key problems to be solved between [38] and this work are completely different. Also, in order to solve MCGDM problem with four tuple information, it builds novel uninorm trust operational laws as well as some new trust aggregating operators (UTWA and UTOWA) and trust propagating operators.

\section{Conclusion}

This article proposes a novel model for group decision making under social network (SN-GDM). To do that, it uses four tuple information to model individual preferences in decision making process, and so it can deal with hesitancy and inconsistency information of experts themselves. Then, it defines the concept of trust decision making space, including trust degree (TS) and knowledge degree (KD), and proposes a ranking order relation for trust function values (TFs), which is used for deriving a final solution in SN-GDM with four tuple information. The operational laws of TFs for uninorm operator are built, and the uninorm trust propagation operator is investigated to propagate the indirect trust relationship between group of experts. It has the advantage of propagating trust and distrust information simultaneously, and then it can maintain the four tuple trust information in the propagating process. After the indirect trust relationship is built, the uninorm trust weighted average (UTWA) operator and the uninorm trust ordered weighted average (UTOWA) operator are developed to aggregate individual trust relationship and produce an order relation of TFs associated to individual expert, and ultimately to obtain a final solution to the SN-GDM. Moreover, the weights associated to experts are derived in a reasonable way as: the higher trusted an expert is the more importance is assigned to the expert.

Nowadays, due to the increase use of social network by citizens, there is an increase interest in dealing with group decision making problems within a social network framework. For example, a group of friends would like to watch a film for which some kind of agreement or consensus is to be reached before deciding on the fill of interest to the group. How to reach consensus within a SN-GDM is a research question we will aim to investigate in future, and in particular we are interested in the developing of trust induced group consensus model and group recommendation method to support a networked group in achieving the common goal for the group.

\section{Acknowledgements}

The authors are very grateful to the anonymous referees for their valuable comments and suggestions. This work was supported by National Natural Science Foundation of China (NSFC) (No.71571166), 
Zhejiang Provincial Natural Science Foundation of China (No.LY15G010003)and Zhejiang Provincial Key Research Base of Humanistic and Social Sciences in Hangzhou Dianzi University (No.ZD01201502), Zhejiang Provincial Qianjiang Talent Foundation of China (No.QJC1402015) and Zhejiang Provincial Social Science Association Foundation of China (2015Z026).

[1] Alonso, S. , Chiclana, F., Herrera, F., Herrera-Viedma, E., Alcala-Fdez, J. and Porcel, C. (2008). A consistency-based procedure to estimate missing pairwise preference values. International Journal of Intelligent Systems 23, 155-175.

[2] Alonso, S., Pérez I. J., Cabrerizo, F. J., and Herrera-Viedma, E. (2013). A linguistic consensus model for web 2.0 communities. Applied Soft Computing 13(1), 149-157.

[3] Atanassov, K. T. (1986). Intuitionistic fuzzy sets. Fuzzy Sets and Systems 20(1), 87-96.

[4] Atanassov, K. T. (2012). On intuitionistic fuzzy sets theory. Springer-Verlag.

[5] Cheng, S. M., and Tsai, B. H. (2015). Autocratic decision making using group recommendations based on the OWA operator and correlation coefficients based on distribution assessments. Information Sciences 290, 106-119.

[6] Chiclana, F., Herrera-Viedma, E., Alonso, S. and Herrera, F. (2009). Cardinal consistency of reciprocal preference relations: a characterization of multiplicative transitivity. IEEE Transactions on Fuzzy Systems 17(1), 14-23.

[7] Chiclana, F., Herrera-Viedma, E., Alonso, S., and Herrera, F. (2008). A Note on the Estimation of Missing Pairwise Preference Values: A Uninorm Consistency Based Method. International Journal of Uncertainty, Fuzziness and Knowledge Based Systems 16, 19-32.

[8] Cornels, C., Lu, J., Guo, X., and Zhang, G. (2007). One-and-only item recommendation with fuzzy logic techniques. Information Sciences 177, 4906-4921.

[9] De Baets, B. and Fodor, J. (1999) Van Melle's combining function in MYCIN is a representable uninorm: an alternative proof. Fuzzy Sets Syst., vol 104, 133-136.

[10] De Cock, M., and Pinheiro Da Silva, P. (2006). A many-valued representation and propagation of trust and distrust. Lecture Notes in Computer Science 3849, 108-113.

[11] Dong, Y. C., and Zhang, H. J. (2014). Multiperson decision making with different preference representation structures: A direct consensus framework and its properties. Knowledge-based Systems $58,45-57$.

[12] Dong, Y. C., Chen, X., and Herrera, F. (2015). Minimizing adjusted simple terms in the consensus reaching process with hesitant linguistic assessments in group decision making. Information Sciences 297, 95-117. 
[13] Dong, Y. C., and Herrera-Viedma, E. (2015). Consistency-driven automatic methodology to set interval numerical scales of 2-tuple linguistic term sets and its use in the linguistic GDM with preference relation. IEEE Transactions on Cybernetics 45, 780-792.

[14] Dong, Y. C., Li, C. C., Xu, Y. F., and Gu, X. (2015). Consensus-based group decision making under multi-granular unbalanced 2-tuple linguistic preference relations. Group Decision and Negotiation 24, 217-242.

[15] Fodor, J. P., Yager,R. R., and Rybalov, H. (1997). Structue of uninorms. International Journal of Uncertainty, Fuzziness and Knowledge Based Systems 5, 411-427.

[16] Hanneman, R. A., and Riddle, M. (2005). Introduction to social network methods. University of California: Riverside.

[17] Herrera-Viedma, E., Alonso, S., Chiclana, F., and Herrera, F. (2007). A consensus model for group decision making with incomplete fuzzy preference relations. IEEE Transactions on Fuzzy Systems 15(5), 863-877.

[18] Herrera-Viedma, E., Cabrerizo, F. J., Kacprzyk, K., and Pedrycz, W. (2014). A review of soft consensus models in a fuzzy environment. Information Fusion 17, 4-13.

[19] Herrera-Viedma, E., Chiclana, F., Herrera, F., and Alonso, S. (2007). Group decision-making model with incomplete fuzzy preference relations based on additive consistency. IEEE Transactions on Systems, Man, and Cybernetics, Part B: Cybernetics 37(1), 176-189.

[20] Kacprzyk, J., Fedrizzi M., and Nurmi, H. (1992). Group decision making and consensus under fuzzy preferences and fuzzy majority. Fuzzy Sets and Systems 49, 21-31.

[21] Klement, E. P., Mesiar, R., and Pap, E. (1996). On the relationship of associative compensatory operators to triangular norms and conorms. International Journal of Uncertainty, Fuzziness and Knowledge Based Systems 4, 129-144.

[22] Li, Y. M, and Lai, C. Y. (2014). A social appraisal mechanism for online purchase decision support in the micro-blogosphere. Decision Support Systems 59, 190-205.

[23] Mata, F. , Pérez, L. G., Zhou, S.M. and Chiclana, F. (2014). Type-1 OWA methodology to consensus reaching processes in multi-granular linguistic contexts. Knowledge-Based Systems 58, $11-22$.

[24] Ölçer, A. İ., and Odabaşi, and A. Y. (2005). A new fuzzy multiple attributive group decision making methodology and its application to propulsion/manoeuvring system selection problem. European Journal of Operational Research 166, 93-114. 
[25] Pérez-Asurmendi, P., and Chiclana, F. (2014). Linguistic majorities with difference in support. Applied Soft Computing 18, 196 - 208.

[26] Rudas, I. J., Pap, E., and Fodos, J. (2013) Information aggregation in intelligent systems: An application oriented approach. Knowledge-Based Systems 38, 3-13.

[27] Scott, H. P. (2000). Social Network Analysis: A Handbook. Sage Publications Ltd: London.

[28] Victor, P. (2010). Trust Networks for Recommender Systems. Ghent University.

[29] Victor, P., Cornelis, C., De Cock, M., and Herrera-Viedma., E. (2011). Practical aggregation operators for gradual trust and distrust. Fuzzy Sets and Systems 184(1), 126-147.

[30] Victor, P., Cornelis, C., De Cock, M., and Pinheiro da Silva., P. (2009). Gradual trust and distrust in recommender systems. Fuzzy Sets and Systems 160(10), 1367-1382.

[31] Wang, J. Q., Li, K. J., and Zhang, H. Y. (2012). Interval-valued intuitionistic fuzzy multi-criteria decision-making approach based on prospect score function. Knowledge-Based Systems 27, 119-215.

[32] Wasserman, S., and Faust, K. (2009). Social Network Analysis: Methods and Applications. Cambridge University Press.

[33] Wu, J., and Chiclana, F. (2012). Non-dominance prioritization methods for intuitionistic and interval-valued intuitionistic preference relations. Expert Systems with Applications 139, 1340913416.

[34] Wu, J., and Chiclana, F. (2014). A risk attitudinal ranking method for interval-valued intuitionistic fuzzy numbers based on novel attitudinal expected score and accuracy functions. Applied Soft Computing 22, 272-286.

[35] Wu, J., and Chiclana, F. (2014). A social network analysis trust-consensus based approach to group decision-making problems with interval-valued fuzzy reciprocal preference relations. Knowledge-Based Systems 59, 97-107.

[36] Wu, J. and Chiclana, F. (2014). Visual information feedback mechanism and attitudinal prioritisation method for group decision making with triangular fuzzy complementary preference relations. Information Sciences 279, 716-736.

[37] Wu, J. and Chiclana, F. (2014). Multiplicative consistency of intuitionistic reciprocal preference relations and its application to missing values estimation and consensus building. Knowledge-Based Systems Volume 71, 187-200.

[38] Wu, J., Chiclana, F., and Herrera-Viedma, E. (2015). Trust based consensus model for social network in an incomplete linguistic information context. Applied Soft Computing 35, 827-839. 
[39] Wu, J., Cao, Q. W., and Zhang, J. L. (2010). Some properties of the induced continuous ordered weighted geometric operators in group decision making. Computers and Industrial Engineering 59, $100-106$.

[40] Wu, J., Li, J. C., Li, H., and Duan, W. Q. (2009). The induced continuous ordered weighted geometric operators and their application in group decision making. Computers and Industrial Engineering 57, 1545-1552.

[41] Wu, Z. B., and Xu, J. P. (2012). A consistency and consensus based decision support model for group decision making with multiplicative preference relations. Decision Support Systems 52, $757-767$.

[42] Xu, J. P., and Shen, F. (2014). A new outranking choice method for group decision making under Atanassovs interval-valued intuitionistic fuzzy environment. Knowledge-Based Systems 70, $177-188$.

[43] Xu, J. P, and Wu, Z. B. (2011). A discrete consensus support model for multiple attribute group decision making. Knowledge-Based Systems 24, 1196-1202.

[44] Xu, J. P, and Wu, Z. B. (2013). A maximizing consensus approach for alternative selection based on uncertain linguistic preference relations. Computers and Industrial Engineering 64, 999-1008.

[45] Xu, Z. S. (2009). An automatic approach to reaching consensus in multiple attribute group decision making. Computers and Industrial Engineering 56, 1369-1374.

[46] Xu, Z. S., and Liao, H. C. (2015). A survey of approaches to decision making with intuitionistic fuzzy preference relations. Knowledge-Based Systems 80, 131-142.

[47] Yager, R. R. (1996). Quantifier guided aggregation using owa operators. International Journal of Intelligent Systems 11(1), 49-73.

[48] Yager, R. R.and Rybalov, A. (1996). Uninorms aggretation operators. Fuzzy Sets Syst., 80, $111-120$.

[49] Zadeh, L. A. (1965). Fuzzy sets. Information and Control 8 (3), 338-357.

[50] Zadeh, L. A. (1983). A computational approach to fuzzy quantifiers in natural languages. Computers and Mathematics with Applications 9(1), 149-184.

[51] Zhang, G. Q., Dong, Y. C., and Xu, Y. F. (2014). Consistency and consensus measures for linguistic preference relations based on distribution assessments. Information Fusion 17, 46-55. 